\title{
Therapeutic Crisis Intervention in Schools (TCI-S): An international exploration of a therapeutic framework to reduce critical incidents and improve teacher and student emotional competence in schools.
}

\begin{tabular}{|r|l|}
\hline Journal: & Journal of Psychologists and Counsellors in Schools \\
\hline Manuscript ID & JGC-PP-2020-0053.R4 \\
\hline Kanuscript Type: & Practitioner Paper \\
\hline Abstract: & $\begin{array}{l}\text { Trauma, Therapeutic, Critical incident framework } \\
\text { the TCI-S framework to reduce critical incidents within the United States } \\
\text { and the United Kingdom school systems are demonstrated through } \\
\text { upskilling school staff in social-emotional and co-regulation skills. It is } \\
\text { suggested that TCI-S has the potential to support students with } \\
\text { behavioural and emotional challenges and increase staff competency to } \\
\text { implement trauma-informed practices that ultimately will reduce critical } \\
\text { incidents. }\end{array}$ \\
\hline & $\begin{array}{l}\text { Critical incident frameworks applied within schools are a means to } \\
\text { support school staff to respond and guide planning to reduce critical } \\
\text { incidents rates. This article explores the significance of the Therapeutic }\end{array}$ \\
\hline
\end{tabular}

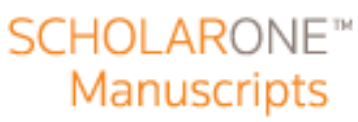


Therapeutic Crisis Intervention in Schools (TCI-S): An international exploration of a therapeutic framework to reduce critical incidents and improve teacher and student emotional competence in schools.

Stella Rodgers* and Sharinaz Hassan**

*Department of Education, School Psychology Services, Western Australia, Australia

**Department of Psychology and Speech Pathology, Curtin University, Western Australia, Australia

\section{Abstract}

Critical incident frameworks applied within schools are a means to support school staff to respond and guide planning to reduce critical incidents rates. This article explores the significance of the Therapeutic Crisis Intervention in Schools (TCI-S) critical incident framework to decrease the prevalence of behavioural critical incidents and to improve staff and student emotional competence in schools. The application of the TCI-S framework to reduce critical incidents within the United States and the United Kingdom school systems are demonstrated through upskilling school staff in social-emotional and co-regulation skills. It is suggested that TCI-S has the potential to support students with behavioural and emotional challenges and increase staff competency to implement trauma-informed practices that ultimately will reduce critical incidents.

\section{Introduction}

In the education profession, more than 1 in 4 Australian teachers suffer from emotional exhaustion once employed and expect to leave the profession within the first five years of teaching (Marshall, 2013). Challenging student behaviour, reactive management strategies from staff, aggression from students and parents, and low morale stemming from toxic school cultures have been identified as contributing factors (WorkCover, 2014). Interestingly, recent research shows that student violence and challenging behaviour in school is strongly associated with childhood trauma and/or childhood stressors (Australian Institute of Family Studies, 2019). This is not to say that all challenging behaviour can be linked back to experiences of trauma. Across education systems internationally, the enactment of policies, directives and procedures to help 'control' challenging student behaviour have been 
commonplace (Sullivan et al., 2014). These approaches have been seen to be either complementary or contradictory to the wellbeing of both students and staff.

Trauma is defined as an event, series of events, or set of circumstances that are experienced as physically or emotionally harmful or life-threatening that may lead to a disruption in overall functioning (SAMSHA, 2014). In children, the typical psychological impacts of trauma include terror, helplessness, stress, learning, attention difficulties, and anxiety (Perry, 2007). These underlying problems can often be masked by external presentations of noncompliance and other challenging behaviour at school. Early trauma can impact brain function, development and emotional regulation, which can lead to mental health disorders including anxiety, depression, substance abuse, posttraumatic stress, and other trauma-related issues in adult life (De Bellis \& Zisk, 2014). There is a lack of empirical knowledge on the exact prevalence rates among Australian children experiencing traumatic events.

Nevertheless, schools continue to report the resulting repetitive violence and challenging pain-based behaviour that presents from these adverse experiences.

Trauma-informed practices (TIP) are models of care and support provision that consider the prevalence of childhood trauma and its subsequent impacts on development, learning, and well- being (Morgan et al., 2015). TIP is an alternative to traditional behaviour-based approaches to understanding and intervening with student challenges, mental health concerns, and problem behaviours (Dorado et al., 2016). Trauma-informed care is used worldwide and across organisations to support young people and adults affected by the impact of adverse life experiences.

Therapeutic Crisis Intervention in Schools (TCI-S) is a critical incident framework that considers a trauma-informed approach to reduce the use of physical restraints, suspensions and improve academic attainments while teaching coping skills with the most vulnerable students (Holden et al., 2012). Cornell University first developed the Therapeutic Crisis Intervention (TCI) crisis prevention and intervention model for residential childcare organizations as part of the Residential Child Care Project (RCCP) in the United States in the early 1980s. The model was developed under a grant from the National Centre on Child Abuse and Neglect and was designed predominantly for residential childcare organizations. Due to the successful outcomes of TCI in this domain, the model branched out to health agencies and into the education field. 
TCI in schools began in the 1980s with larger campus types of residential treatment facilities, such as Waterford Country School located in the state of Connecticut. Several large school districts in the US used TCI in the 1990s along with many special education centres. TCI-S currently being applied internationally in schools and is gathering an evidence base for demonstrating improvements across student mental health, academic engagement and the prevalence of critical incidents. These are the goals that guide TCI-S practice in schools; i) prevention of crises from occurring, ii) assisting teachers in de-escalating and managing potential crises, iii) reducing physical restraints, iv) learning constructive ways for staff to handle stressful situations, v) teaching student coping skills, vi) developing a learning circle within organisations, and viii) using contemporary behaviour and trauma research to inform practices (The Therapeutic Crisis Intervention for Schools, [TCI-S], 2012). A significant difference to other critical incident frameworks is that TCI-S teaches school staff about emotional competence. By training teachers to view students' behaviour through a traumainformed lens, their responses to the challenging behaviour can address the student's underlying needs.

In Australia, TCI has been offered within the clinical setting, and in Western Australia particularly, it is implemented within Child Protection and Family Support (CPFS), Department of Health and Child and Adolescent Mental Health Services (CAMHS). In 2016, TCI-S was introduced into the School of Specialised Educational Needs: Behaviour and Engagement (SSEN:BE) centres in the Western Australian Department of Education. The Department of Health's Family Pathways service and Adolescent Unit have also introduced TCI in suburban clinics and hence, TCI is already broadening its reach within this state.

\section{TCI-S Internationally}

Research has shown that schools in America and the United Kingdom have been applying the TCI-S framework to address issues of violence and poor student mental health. Figure 1 depicts the practical TCI-S five domains to successfully implement TCI-S within a school system. The five domains are outlined in detail. 


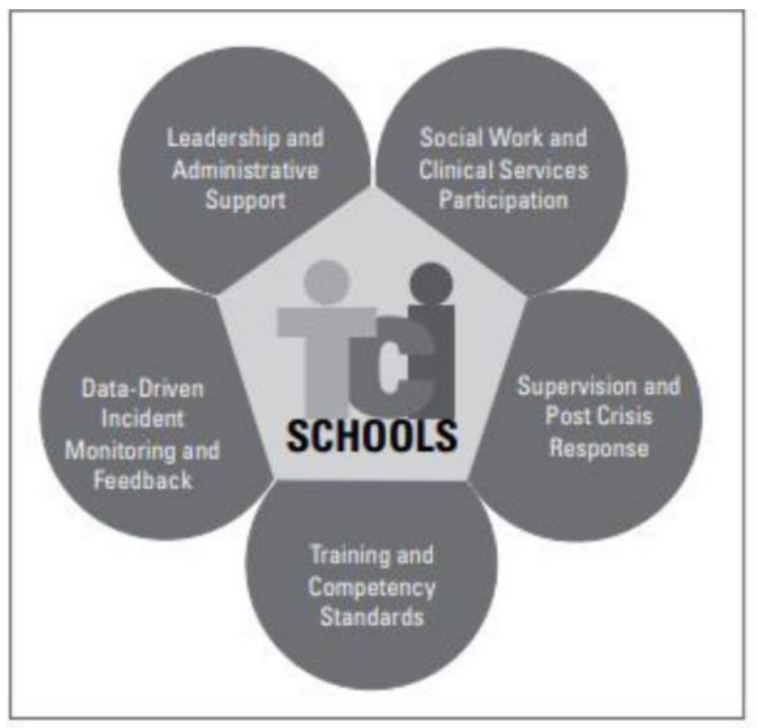

Figure 1: Five domains for effective TCI -S implementation in schools. From "Therapeutic Crisis Intervention System for Schools Information Bulletin”, The Residential Child Care Project College of Human Ecology Cornell University, Ithaca, NY USA.

Copyright (2012) by Bronfenbenner Center for Translational Research. Reprinted with permission.

\section{Leadership and Administration support}

The domain of Leadership and Administrative support aims to develop a whole-school approach or framework to respond to challenging student behaviour. Promoting psychological health, applying a shared systemic response to challenging behaviour, and implementing a relational repair model for students and staff following critical incidents are the key goals of this domain (TCI-S, 2012). For schools, the leadership commitment begins with both the school leadership and regional support to provide the school with guidance to implement the TCI-S crisis management system. Leadership teams and administration support are fully informed about the TCI-S crisis prevention and management system, and this will lead school staff to provide a consistent response to crises. Within the school setting, the TCI-S framework represents a standard communication of crisis procedures, policies, and practices in the organisation (TCI-S, 2012). School staff are educated on how to prevent and de-escalate student challenging behaviour and teach coping skills. The TCI-S framework is essential in developing and maintaining a safe and supportive school culture that promotes academic growth and student wellbeing. Leaders can promote a school culture that 
establishes an environment where students can learn by valuing developmentally appropriate practice above control (TCI-S, 2012).

Several schools were visited and interviewed by the first author in the New York and Connecticut State in the US (specifically Waterford Country School and Red Jacket School, a mainstream middle school). These schools had involved their entire school staff trained in TCI -S, including the gardeners and cleaners. These schools had two 'champion' staff, trained as TCI -S trainers, who then trained the remainder of the school staff.

The Board of Co-Operative Educational Services (BOCES) in Newark also had all of their staff trained in TCI-S and this was led by their school Director. BOCES is a service in the US that provides shared educational programs to school districts within each state. The BOCES Director provided a top-down approach to leading and supporting TCI-S implementation alongside the district Principals and Principal Superintendents. The mainstream schools in these districts also adopted combined programs including Positive Behaviour in Schools (PBiS) and social-emotional programs in combination with TCI-S, which proved to further reinforce TCI-S strategies. Waterford Country School (WCS) also adopted CARE (Children And Residential Experiences) specific to their context. CARE is a multi-level program model for improving services for children in out-of-home care as seen in WCS.

The leadership team at WCS consistently allocate time for staff members to be released from teaching duty to respond to critical incidents. The TCI-S strategies of co-regulation and emotional first aid have helped the staff to assist students to return to their emotional baseline. Using one of the strategies of TCI-S, the Life Space Interview (LSI) technique, reparation of student-teacher relationships was observed. The LSI is an approach used to change behaviour patterns of students. It is used as a crisis intervention technique during the recovery phase of a behaviour escalation in which a student's behaviour is discussed with the student at the time of the problem's occurrence, or within the 'life space'.

After the implementation of TCI-S, school staff at WCS noted that they experienced more job satisfaction and that more students remained in the school program rather than being suspended for challenging behaviour. "When teachers could repair relationships with students before the end of the day, they reported a significant positive impact on the following day's events" as quoted by a residential teacher as noted in the WCS interview notes of the visit. 


\section{Social Work and Clinical Services Participation}

Social work and Clinical Services play an essential role in overseeing and monitoring staff responses to students in crisis (TCI-S, 2012). In the US, the utilisation of social work support is similar to the use of school psychology services in Australia for supporting the TCI-S system. The development of Individual Documented Plans or Individual Crisis Management Plans in consultation with clinical services for responding to behaviour escalations is critical for staff to minimise the need for physical restraint (TCI-S, 2012).

The facilitation of ongoing reviews of students' Individual Documented Plans following behaviour escalations assists staff to develop more effective ways to prevent and intervene with student's high-risk behaviour (Holden et al., 2012). The revisions are informed by the data generated from incident reports. Thus, planning cycles of review are set in place with a team of relevant clinical staff in order to meet student needs and support behaviour change.

At WCS, it was evident that the social workers/school psychologists were working as 'Instructional coaches' of TCI-S. School psychologists have the capacity to review behaviour incidents with school staff and support in reviewing documentation planning and coaching of TCI-S strategies. School psychologists alongside Occupational Therapists, Speech Therapists, School Nurses, Psychiatrists and nominated school staff attend Care-Team meetings once per term to review every child's documented plan. In the US, schools apply for grants to broker clinical services to work on school sites while others resource within their own budgets. As such, TCI for Families (TCI-F) is a specialised branch of TCI-S that coach parents in strategies for the home environment. The facilitation of TCI-F was seen to be used effectively in Klingberg Family Centre (KFC). KFC resides in the city of Hartford within the US state of Connecticut. KFC is a private, not for profit multi-service agency that assists families affected by trauma and houses the Raymond Hill School for students with special needs. The TCI-F link allowed for a holistic approach to supporting these students both at school and home.

\section{Supervision and Post-Crisis Response}

Frequent and ongoing supportive staff supervision, mentoring, and coaching is essential for creating and sustaining a school's ability to reduce the need for physical restraint (TCI-S, 2012). The facilitation of weekly reflective and supportive supervision is built into the 
implementation and ongoing monitoring of the TCI-S crisis management system. School leadership provides the time to prioritize reflective supervision of their school staff as a routine practice to reduce the potential for teacher burnout. Teachers meet once a week for up to an hour at a time to work through teaching goals. A post-crisis response system is also set up in the TCI-S framework that allows for all students and staff to receive immediate support and debriefing following a crisis (TCI-S, 2012). Following a critical incident, all staff involved can deconstruct the crisis to develop strategies for intervening differently in the future. The post- crises response system is built on the LSI format. It allows for both an emotional drain-off followed by an operational debrief to interrupt the re-enactment of trauma and improve responses to student behaviour after every incident.

During the US school visits, it was observed that staff had individual escalation profiles on their desks for the student's they supported. At WCS, these visuals were used to assist staff themselves in using self-awareness and emotional competence to regulate their own behaviour in response to student behaviour. Students also had their own safety plan and escalation profile that they developed to identify when they needed support to regulate their behaviour. After an escalation of behaviour, students were debriefed using the LSI model by an appropriate adult on the same day as the crisis. Students would become so used to the routine that they would say, "I'm ready to process now" to the adults. The critical incident debriefing process is a core practice and assists teachers in being responsive to regulate and contain dysregulated students.

\section{Training and Competency Standards}

Training is a key element in the TCI-S framework. Schools choose training for staff that is reflective of their school's context to develop and improve academic success and engagement. The scope of training for a school to maintain TCI-S understandings includes Professional Learning (PL) in such areas as disability, trauma, mental health and classroom behaviour strategies (TCI-S, 2012). These trainings are at the discretion of the school, and leadership allows resourcing and time for the PL to be prioritised.

The TCI-S training is run over three days with a minimum of 21 hours. TCI-S trainers are required to attend a Cornell University sponsored Update and pass testing requirements at least every two years in order to maintain their certification to continue to provide training for their own school staff. Training for staff to refresh TCI-S skills is required at a rate of three 
hours every three months in-house. These refreshers can be creatively organised into school staff meetings. Refreshers are designed to give staff the opportunity to practise de-escalation skills, the LSI, emotional first aid and crisis co-regulation. Documentation of these training events and assessment of staff level of competency is critical in order to maintain the TCI-S system.

\section{Data-driven Incident Monitoring and Feedback}

Documentation, data analysis, and feedback to all levels of staff teams are an essential part of physical restraint reduction efforts and reducing mental health problems with students (TCIS, 2012). The TCI-S documentation reflects changes in data that contribute to the effectiveness of the TCI-S system. This documentation and monitoring system allow for the school to review incidents and make decisions about individual and organisational practice to recommend corrective action (TCI-S, 2012).

In Scotland at the residential Hillside School of Fife, the impetus for change was the number of physical restraints being used on-site with students who were dysregulated and/or physically aggressive. Over three years, the school reduced the number of restraints of students aged 8-18 years from several per day to two to three per week. As an additional positive effect, students attending WCS demonstrated improvements in overall mental health. In particular, after 6 months of TCI-S combined with CARE, staff at WCS observed a reduction in the number of psychotropic medications being prescribed to individual students (Smith et al., 2019). When explored further, WCS reported student wellbeing was improved because of the new communication style used by the WCS' staff to the clinical staff. An example included using terminology such as 'the student was demonstrating pain-based behaviour' as opposed to 'the student was being controlling and manipulative'. Students observed the trauma-informed care practise instead of the pathology-driven description of behaviour, and this positively impacted all aspects of the student's life.

Data collected across schools included attendance, academic attainment, student readiness for change, police involvement to the school, staff burnout and retention as well as the number of detentions and suspension data. The use of data collection tools differed across schools which included; Social and Emotional Learning pre-screening surveys and assessment, the Strengths and Difficulties Questionnaire (SDQs) for parents and students (pre and post) and the ARTIC 
tool (Assessment of Staff Attitudes to Trauma-Informed Care). The use of data has allowed these schools to inform planning and strategic direction with their students.

\section{TCI-S Limitations}

It is important to note that while observing the positive outcomes of the qualitative research with TCI-S internationally, there were limitations identified with application in schools. It has been demonstrated that TCI-S is unlikely to be a successful model if leadership do not take ownership and provide resources for school staff to implement. If a dedicated team of school staff decide to train in TCI-S but the impetus has not come from leadership in a topdown approach, TCI-S, while it might have some effect for that team, can lose its rigour and be utilised as a training program rather than a systemic framework. By implementing TCI-S in this way, not all staff are likely to remain consistent in the systemic approach and impact for students can be lost.

Time and resource commitment are an imperative component of the TCI-S system. When schools are unable to prioritize training time and staff resourcing to manage critical incidents,

TCI-S can lose its integrity. The time and resourcing allocated from leadership is required at times, to be prioritized over academic teaching. This shift in lens can be difficult for some schools when governed by outcomes-based education approaches and calls for student performance accountability.

The TCI-S emotional competence training appeared to be useful for staff and has been reported to have had an indirect effect on students' challenging behaviour. However, the dosage treatment for school staff and students needs to be balanced to ensure inclusive outcomes for all involved. TCI-S implementation has not yet been investigated systematically using randomised controlled trials and longitudinal research is also lacking.

\section{Conclusion and Implications}

Implementation of TCI-S in the US and UK show an increased ability on the part of staff to manage and prevent crises, as well as to assist students to learn new ways of coping. The most striking findings of the TCI-S research has been that TCI-S works as an emotional competence training framework for staff. The implications for staff with strong emotional competence have been observed to influence a positive safe school culture. The other unique 
finding with the TCI-S system is that students who, in the past, demonstrated unhelpful behaviour to get their needs met have been taught new ways to respond and interact through day-by-day interactions with emotionally competent adults. As a result, there have been observed reductions in violent and emotionally driven behaviour responses in schools.

The implications of these findings suggest:

1. the need and benefit for leadership to endorse a top-down approach to implementing a framework such as TCI-S in schools;

2. TCI-S works best when combined with a program inside the framework such as CARE or a social and emotional program; for example, in the Australian context Berry Street Model, Promoting Alternative Thinking Strategies (PAThS), Aussie Optimism;

3. the need for post-crisis supervision and debrief processes for school staff following every critical incident;

4. the essential link with family and clinical services in schools to support at-risk students; and

5. the importance of ongoing education of school staff about trauma and traumainformed practices to equip schools with strategies and resources to teach coping skills to students unable to function effectively in mainstream school settings.

In Australia, TCI has been offered within the clinical setting, and in Western Australia particularly, it is implemented within Child Protection and Family Support (CPFS), Department of Health and Child and Adolescent Mental Health Services (CAMHS). In 2016, TCI-S was introduced into the School of Specialised Educational Needs: Behaviour and Engagement (SSEN:BE) Engagement Centres in the Western Australian Department of Education. The Department of Health's Family Pathways service and Adolescent Unit have also introduced TCI in suburban clinics and hence, TCI is already broadening its reach within this state. Based on these findings and promising results, TCI-S training and implementation in schools is at an early stage in Australia and in particular in Western Australia. The results are still being monitored about staff emotional competence and the compounding impact on challenging behaviour.

Given that TCI-S has been successfully implemented within schools in the US and UK, these findings warrant conducting empirical studies in Australian schools. Factors to be considered are the effect of moderating variables; for example, boys and girls may show differential 
responses to the TCI-S intervention. The influence of mediating variables should also be taken into account. These include improved parent/carer supervision and relations with youth, and integration with evidence-based social-emotional interventions. Furthermore, a comprehensive analysis to investigate the predictor factors is highly advocated. These are variables that influence the likelihood of an outcome for a given treatment. Predictor variables may identify whether the treatment effect is stronger under certain conditions; for example, higher or lower levels of evidence- based program support.

A hypothetical question for further exploration may be to examine is whether TCI-S functions as a stand-alone model, or a model that works in combination with established social-emotional programs that will more likely augment the TCI-S goals? Further study is required to determine the tailored mechanism for effective implementation of traumainformed practices within the school setting. The anticipated findings will help to distinguish the uniqueness of TCI-S compared to other critical incident frameworks with a traumainformed lens and to aid in the future direction of TCI-S.

This paper was the result of a funded research project, developed by the Anika Foundation to raise funds for the purposes of supporting research into adolescent depression and suicide.

The Anika Foundation provides an opportunity for applicants in Australia to make a difference, in an area that affects at least 1 in 4 families. The aim of this paper is to distribute the research findings at a national level to promote awareness into effective trauma-informed practices for schools to support this invaluable venture. 


\section{References}

Australian Government: Australian Institute of Family Studies. (2019). Retrieved from https://aifs.gov.au/cfca/publications/effect-trauma-brain-development-children

Bedi, G., \& Goddard, C. (2007). Intimate Partner Violence: What are the impacts on children? Australian Psychologist, 42(1), 66-77. doi: 10.1080/00050060600726296

Ford, J. D., \& Courtois, C. A. (2009). Defining and understanding complex trauma and complex traumatic stress disorders. Treating complex traumatic disorder: An evidence-based guide. In. New York, US: Guilford Press.

De Bellis, M. D., \& Zisk, A. (2014). The biological effects of childhood trauma. Child and Adolescent psychiatric clinics of North America, 23(2), 185-vii. doi: 10.1016/j.chc.2014.01.002

Dorodo, J., Martinez, M., McArthur, L., \& Leibovitz, T. (2016). Healthy Environments and Response to Trauma in Schools (HEARTS): A whole-school, multi-level, prevention and intervention program for creating trauma-informed, safe and supportive schools. School Mental Health, 8, 163-176. doi: 10.1007/s12310-016-9177-0

Holden, M., Holden, J. \& the TCI-S Instructors of the Residential Child Care Project. (2012). Therapeutic Crisis Intervention, Reference guide ( $1^{\text {st }} e d$.). Ithaca, NY: Cornell University.

Marshall, K. (2013). Burnout hits one in four teachers. Retrieved from https://www.theage.com.au/national/victoria/burnout-hits-one-in-four-teachers20131005-2v13y.html

Morgan, A., Pendergast, D., Brown, R., \& Heck, D. (2015). Relational ways of being an educator: Trauma-informed practice supporting disenfranchised young people. International Journal of Inclusive Education, 19(10), 1037-1051. doi: 10.1080/13603116.2015.1035344

Perry, B. (2007). Stress, Trauma and Post-Traumatic Stress Disorders in Children. Retrieved from https://childtrauma.org/wp-content/uploads/2013/11/PTSD_Caregivers.pdf 
Record-Lemon, R. M., \& Buchanan, M. J. (2017). Trauma-Informed Practices in Schools: A narrative Literature Review. Canadian Journal of Counselling and Psychotherapy, 51(4), 286-305.

Substance Abuse and Mental Health Services Administration (SAMHA). (2014). SAMHAS'S Concept of Trauma and Guidance for a Trauma-Informed Approach. Rockville, MD Retrieved from https://store.samhsa.gov/system/files/sma14-4884.pdf.

Smith, E. G., Nunno, M. A., Martin, W. R., \& Sellers, D. E. (2019, April). Does the CARE Program Model Reduce Use of Psychotropic Medications in Residential Care? Poster presented at the 63rd Annual Conference of the Association of Children's Residential Centers, New Orleans, LA.

Sullivan, A., Johnson, B., Owens, L., Conway, B., Lucas, B., \& Baak, M. (2014). Behaviour at School Study (A framework for developing and enacting humane behaviour policies and practices in schools). Australian Research Council Linkage Grant. Retrieved from

https://www.bass.edu.au/files/1914/1565/8195/Framework_for_Humane_Behaviour. pdf

The Therapeutic Crisis Intervention System for Schools (TCI -S). (2012). Information Bulletin. Ithaca, NY: Cornell University. Retrieved from https://rccp.cornell.edu/_assets/TCIS_SYSTEM_BULLETIN.pdf

WorkCover. (2014). Annual Report 2013-2014. Retrieved from https://www.workcover.wa.gov.au/wp-content/uploads/2014/09/Final-DesignInteractive-Web-PDF-WorkCoverWA-Annual-Report2013_2014_Interactive_WholeReport_FINAL_v2.pdf 